\title{
A consensus research agenda for optimising nasal drug delivery
}

Ben Forbes, Rene Bommer, Jonathan Goole, Marie Hellfritzsch, Wilbur De Kruijf, Pierre Lambert, Grazia Caivano, Alain Regard, Francesca Schiaretti, Marie Trenkel, Laurent Vecellio, Gerallt Williams, Fabio Sonvico and Regina Scherließ

\section{QUERY SHEET}

This page lists questions we have about your paper. The numbers displayed at left are hyperlinked to the location of the query in your paper.

The title and author names are listed on this sheet as they will be published, both on your paper and on the Table of Contents. Please review and ensure the information is correct and advise us if any changes need to be made. In addition, please review your paper as a whole for typographical and essential corrections.

Your PDF proof has been enabled so that you can comment on the proof directly using Adobe Acrobat. For further information on marking corrections using Acrobat, please visit http://journalauthors.tandf.co.uk/production/acrobat.asp; https://authorservices.taylorandfrancis.com/how-to-correct-proofs-with-adobe/

The CrossRef database (www.crossref.org/) has been used to validate the references. Changes resulting from mismatches are tracked in red font.

\section{AUTHOR QUERIES}

Q1 Please note that the ORCID for Fabio Sonvico has been created from information provided through Manuscript. Please correct if this is inaccurate.

Q2 Please note that the ORCID for Regina Scherließ has been created from information provided through Manuscript. Please correct if this is inaccurate.

Q3 Please provide missing Department for the affiliation [a,b,e,f,g,h,i].

Q4 Please provide missing city for the affifliation " $\mathrm{e}$ ".

Q5 Please check whether the inserted Keywords are correct.

Q6 The journal follows American spelling. If the term "analyses" is acting as a verb here, and in subsequent instances if any, please change it to "analyzes" to match with the journal style.

Q7 The PubMed (http://www.ncbi.nlm.nih.gov/pubmed) and CrossRef (www.crossref.org/) databases have been used to validate the references. Mismatches between the original manuscript and PubMed or CrossRef are tracked in red font. Please provide a revision if the change is incorrect. Do not comment on correct changes. 


\title{
A consensus research agenda for optimising nasal drug delivery
}

\author{
Ben Forbes ${ }^{a}$, Rene Bommer ${ }^{b}$, Jonathan Goole ${ }^{c}$, Marie Hellfritzsch ${ }^{d}$, Wilbur De Kruijfe, Pierre Lambert ${ }^{c}$, Grazia Caivanof, \\ Alain Regard ${ }^{g}$, Francesca Schiarettif, Marie Trenkel ${ }^{d}$, Laurent Vecelliog,h, Gerallt Williamsi, Fabio Sonvico (i) \\ and Regina Scherließ (1) ${ }^{d}$
}

aKing's College London, Institute of Pharmaceutical Science, London, UK; 'bPharmAccel Consulting, Moos, Germany; 'Laboratory of Pharmaceutics and Biopharmaceutics, Université libre de Bruxelles, Brussels, Belgium; 'Department of Pharmaceutics and Biopharmaceutics, Kiel University, Kiel,

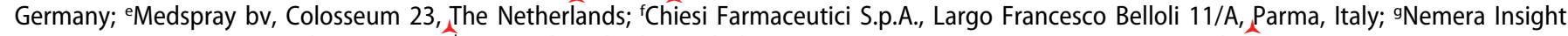
Innovation Center, La Verpilière, France; ${ }^{h}$ Centre d'étude des pathologies respiratoires (CEPR), UMR, Université de Tours, INSERM, Tours, France; 'Aptar, Pharma, Le Vaudreuil, France; 'Biopharmanet TEC, University of Parma, Parma, Italy

\section{ABSTRACT}

Nasal drug delivery has specific challenges which are distinct from oral inhalation, alongside which it is often considered. The next generation of nasal products will be required to deliver new classes of molecule, e.g. vaccines, biologics and drugs with action in the brain or sinuses, to local and systemic therapeutic targets. Innovations and new tools/knowledge are required to design products to deliver these therapeutic agents to the right target at the right time in the right patients. We report the outcomes of an expert meeting convened to consider gaps in knowledge and unmet research needs in terms of (i) formulation and devices, (ii) meaningful product characterization and modeling, (iii) opportunities to modify absorption and clearance. Important research questions were identified in the areas of device and formulation innovation, critical quality attributes for different nasal products, development of nasal casts for drug deposition studies, improved experimental models, the use of simulations and nasal delivery in special populations. We offer these questions as a stimulus to research and suggest that they might be addressed most effectively by collaborative research endeavors.

\section{ARTICLE HISTORY}

Received 28 September 2019

Accepted 8 January 2020

\section{KEYWORDS}

Biopharmaceutics; nose-tobrain; vaccine; sinus; biologics; formulation; device; deposition; nasal cast

\section{Introduction}

Nasal drug delivery has several specific challenges which are distinct from oral inhalation, alongside which it is often considered. An expert meeting was convened on 15 May 2019 to scope the future needs of nasal delivery. The meeting, jointly designed by Professors Sonvico, Scherließ and Forbes, was held at the Department of Pharmaceutics and Biopharmaceutics of Kiel University in Germany and attended by expert academic and industrial associates who included drug delivery scientists, device designers and product developers (listed as coauthors). Deliberations focused on (i) formulation and device needs, (ii) meaningful product characterization and modeling, (iii) influencing absorption and clearance.

Nasal administration is often associated with prescribed and over-the-counter medicinal products for the management of ailments such as nasal congestion, common cold symptoms, seasonal allergies or more persistent conditions such as chronic sinusitis. These are treated with drug solutions or suspensions, typically using a hand-operated metered dose pump that delivers a conical spray upon actuation into the nasal cavities. This simple approach and the ease of administration lends itself to regulatory approaches focused on device and formulation.

The nose offers easy access to a highly vascularized and relatively permeable mucosa, making it attractive not only for topical treatment of local diseases but also for systemic drug delivery. The presence of immunocompetent cells and the direct access to the brain via the olfactory region are auspicious as special target sites for needle-free vaccination or central nervous system targeting. However, factors related to the physiological function of the nose and special needs of new drug candidates are hurdles on the way to optimized nasal drug delivery. Nasal delivery notably provides successful noninvasive systemic delivery for a variety of peptide drugs. Desmopressin was the first nasal peptide product in 1978, quickly followed by calcitonin, buserelin, naferelin and oxytocin [1]. After a period of latency, new applications are now gathering momentum, including (i) vaccination [2], e.g. FluMist $^{\oplus}$ the nasal influenza vaccine, and (ii) drug delivery to the brain [3], e.g. naloxone for emergency reversal of opioid overdose $\left(\mathrm{Narcan}^{\oplus}\right)$ and the recently approved potential blockbuster product esketamine nasal spray (Spravato ${ }^{\odot}$ ) for treatment resistant depression. Illustrative examples of nasal delivery systems in clinical trials include low-dose oxytocin for effects on social-cognitive behavior [4] and insulin for effects on cognition and Alzheimer's disease [5]. There are too many molecules and therapies currently in the pre-clinical and clinical development pipeline to summarize fully in this article; the interested readers are referred to dedicated databases, such as Pharmacircle. There are also multiple patents in the nasal drug 
delivery area, but this too is a complex and specialist area beyond the scope of this article.

75 More challenging drug candidates like water insoluble compounds and degradation-sensitive proteins require more sophisticated formulation and delivery approaches. Furthermore, it is desirable to control deposition, absorptive and non-absorptive clearance by tailored adaption of formulation and/or device, 80 while considering the variability in the entire patient population from infant to geriatric. Meaningful and biorelevant prediction models for deposition, clearance and absorption are needed in which to assess the efficacy of new nasal drug delivery approaches.

The purpose of the meeting was to establish a focus group

85 to pave the way for future development of nasal products by identifying and addressing technical and regulatory hurdles hampering the development of innovative nasal products. This is easier in the precompetitive space where open innovation is possible [6]. If a consensus can be agreed regarding the most

90 important research questions, then expertise can be combined in collaborative efforts to understand better how to optimize, nasal products.

\section{Formulations and delivery devices}

A variety of devices and formulations are well established for

95 nasal delivery (reviewed elsewhere; see [7-10]). Liquid formulations dominate the market and are delivered as drops, pump or propellant-driven sprays, or by nebulization. Powders are generally delivered by devices that spray the powder or require insufflation by the patient. Spray characteristics,

100 deposition, mucociliary clearance, dissolution and absorption strongly affect the performance of nasal drug delivery systems. All of these steps can be influenced by formulation and device design. While the formulation is often key to performance, the device supports an optimized formulation and these compo105 nents should not be considered in isolation. In principle, products for nasal drug delivery should be as simple as possible to reduce failure modes, but there are scenarios where high technology concepts may offer advantages, e.g. absorption enhancement in systemic delivery, targeted deposition in

110 nose-to-brain delivery and adjuvants for nasal vaccination. The ideal attributes of a nasal spray are efficient deposition in the nasal cavity, reproducibility, robustness, tolerability and low respirable fraction [11-13]. The dependence of nasal deposition on aspects of the spray plume is poorly defined

115 as it is technically difficult to vary properties such as droplet size distribution, droplet velocity and spray cone angle systematically and human factors are also a big influence.

Formulation is a critical determinant of product performance and may be the dominant factor for therapeutic efficiency [11]. The critical quality attributes for nasal formulations are not well established, but different pharmacokinetics or clinical outcomes can be influenced by the use of powders versus solutions, different excipients and delivered volume/ mass. A possible strategy to protect the drug from enzymatic 125 degradation may be the forming of protective shells, such as liposomes or micelles, or the addition of enzyme inhibitors and the addition of mucoadhesive agents may enhance absorption by prolonging the residence time of the formulation in the nose $[13,14]$. For powders, changing the particle size distribution or applying particle engineering can influence deposition and dissolution [12]. For wet nasal sprays changing droplet size distribution is more difficult due to limitations of typical swirl spray nozzles.

A better understanding of the local effects of excipients and formulation strategies in the nasal environment is required to precisely control the outcomes. In this regard, marketing applications often include quality-by-design and design-of-experiments analyses, but these are not public data and the effective extent and focus of these investigations has not been standardized. Wide ranges of variables may be tested to evaluate their impact on product performance in vitro - but it is unclear how and to what extent this relates to in vivo performance. Some critical product attributes are likely to be product-specific, whereas others may be more generalizable. Defining these attributes is pivotal to guiding formulation and device optimization in product development and developing 'best practice' for product characterization. An aspect often overlooked is the sensory impact of formulations including taste, odor and somatosensory perception (in particular for powders). While liquids may drip down the esophagus and lead to bad tastes, powders may cause greater sensory irritations [15]. The evaluation of sensory effects of nasal formulations is underestimated until now and masking of unpleasant tastes, odors and sensation in nasal formulations is an unworked field for research. Sensory properties can sometimes be harnessed for positive effects, although blinding in clinical studies can be compromised.

As supportive and inseparable parts of nasal drug delivery systems, the device design and functionality are essential. The impact of different devices on delivered dose and site of deposition (e.g. reservoir vs single dose) and their use by the patient (angle, actuation force, inhalation maneuver) compared to formulation variables (powder or solution) is not understood fully. Devices may be designed to target a specific site within the nasal cavity with the aim of optimizing pharmacokinetics. Device, formulation and patient-related variables influence regional deposition and several attempts have been made to model this in view of understanding and predicting drug deposition in the nasal cavity [16]. There are instances when special product designs may be required for particular delivery needs, e.g. mucosal, sinus, systemic or nose-to-brain delivery, or delivered substance, e.g. small molecule, biologic or vaccine.

The compliance of patients may be encouraged with robust and easy to use devices and minimal device-related sensory effects. High-speed impaction, low temperature or direct contact of the device tip with the particularly sensitive anterior part of nasal mucosa cause unpleasant sensations which need to be avoided [8]. The development and evaluation of gentle devices, especially for children, is therefore desirable.

\section{Product characterization,}

Comprehensive in vitro quality and performance requirements for nasal products include physico-chemical properties of the formulation such as $\mathrm{pH}$, osmolarity and viscosity, device extractables and leachables, delivered dose uniformity through container life, device robustness, spray pattern and 
plume geometry $[7,17,18]$. Human factors guidelines [19] attempt to account for usability and variation introduced by patient-device interaction. Variables include penetration depth, angle, head orientation, actuation force, sniffing/inhalation maneuver, counter and nostril closure. Taking these into account early avoids problems in the clinic and allows a product development 'story' to be developed, although it is unclear whether there are nasal-specific human factors and what these might be.

Formulations are characterized according to product type $[12,20]$. Liquid formulations require a focus on API solubility, viscosity, surface tension and density. For particle/droplet size produced by nasal sprays, the emphasis is on the containment of inhalable aerosols, with a requirement to determine the percentage of droplets below $10 \mu \mathrm{m}$. In contrast, aerodynamic particle size distribution is required for orally inhaled products and several non-compendial tests are available, often aimed at more biorelevant characterization of product performance. In contrast to nasal sprays, nasal nebulizers produce particle of much lower size, predominately less than $5 \mu \mathrm{m}$, however, there is no standard or regulation about nasal nebulizers. When the API is delivered as a solid, e.g. in powders or suspensions, the physical and chemical properties of the particles should be determined and drug dissolution in physiological conditions may become an important

210 consideration. Some tests for nasal products such as spray pattern and plume geometry may be of limited biological relevance, although they may be useful for regulatory or quality control purposes. Some locally-acting formulations are sufficiently straightforward that BE studies can be waived, e.g. mometasone

215 furoate nasal suspension spray [21]. Excipients and physicochemical properties (volume, osmolarity, viscosity) can effect dissolution rate, which may vary according to the environment of the local deposition site in the nasal cavity. Dissolution is not generally considered to be clinically significant for nasal products and is little researched - there are no sustained release nasal spray products. However, despite similar conclusions being reached for orally inhaled drug products in 2012 [22], there is currently a high level of interest in the dissolution of aerosol medicines for oral inhalation.

Computational fluid dynamics (CFD) provides a useful research tool, although predictions of in vivo deposition patterns require experimental verification. Nor is the relationship between spray velocity and in vivo deposition fully elucidated. The US FDA directs nasal medicinal product developers toward deposition studies using casts and dissolution. It is still unclear what the impact of dissolution is for current suspension/powder products. A variety of casts have been developed for predicting regional deposition, analogous to the more advanced casts of the throat/lungs that are being used for pulmonary drug delivery [13] and similar questions arise regarding the need for idealized casts, or casts that reflect sub-populations of patient anatomy or disease. There is little validation of nasal cast models [23], and it has been proposed that biorelevance could be improved by using materials being able to better mimic physiological conditions, such as softer, expandable materials with a flexible nasal valve, or by introducing cells or mucus to reflect the surface characteristics of the nasal mucosa.

\section{Drug retention and absorption in the nasal cavity}

To optimize, nasal drug delivery, the device and formulation must be designed to overcome the physiological constraints on drug delivery (Figure 1). It is difficult to model the interplay of deposition, clearance and absorption that determines local exposure and systemic pharmacokinetics in vitro. The nasal epithelium is generally considered to be less restrictive compared to the gastrointestinal tract, but drug permeability may vary in different regions of the nasal cavity (olfactory, vestibule, turbinates, septum), between species and/or individuals and in pathological conditions, but it is unknown to what extent such variations may impact the pharmacokinetics and clinical effects compared to device and formulation factors. For example, the presence and impact of transporters is unclear. Biorelevant models to evaluate

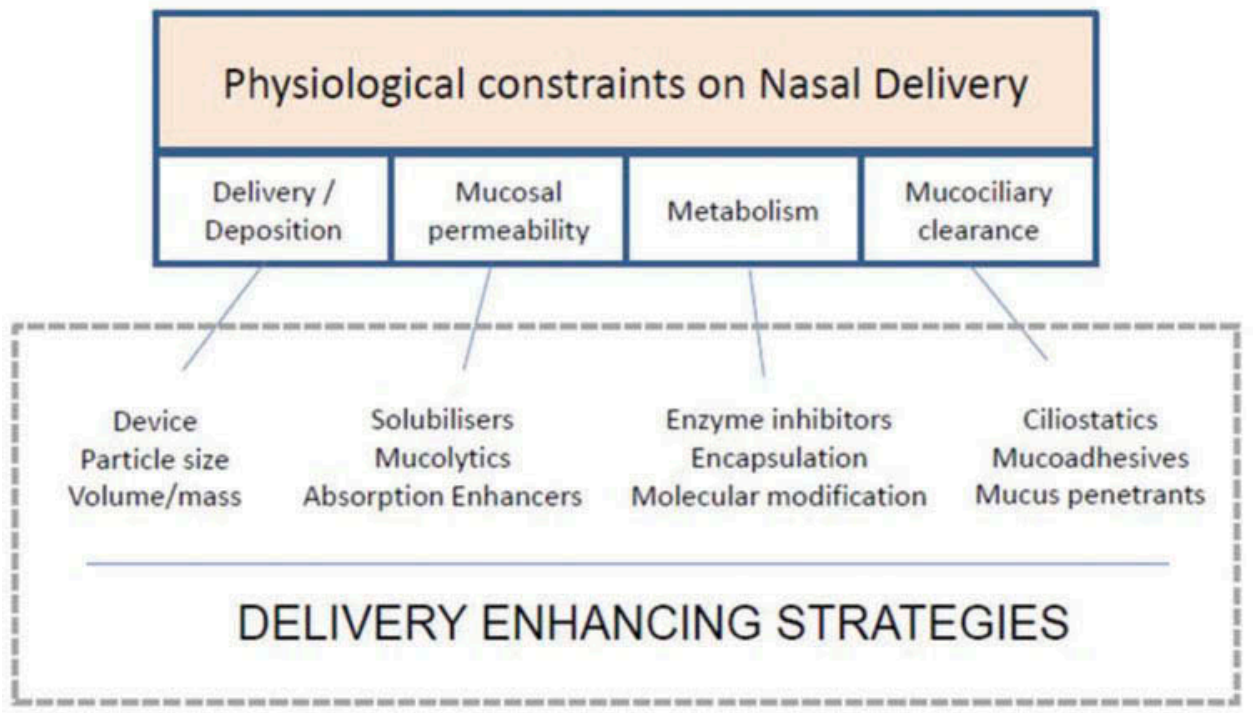

Figure 1. To optimizę nasal drug delivery, the device and formulation must be designed to overcome the physiological constraints on drug delivery [adapted from Ref 24]. 
permeability or dissolution in simulated nasal environments have been developed that can guide the development of bioequivalent products and tackle challenges related to novel products such as poor water solubility, high dose, controlled release formulation, biotechnological products and systemic delivery of actives. Experimental systems for studying drug transport in the airways include cell lines, e.g. Calu-3 [24] or RPMI 2650 [26], 265 primary cells [27] and reconstructed epithelia [28]. Neuronepithelial cocultures are under development to simulate the nose-to-brain route [29] as an alternative to ex vivo olfactory tissue which is difficult to use. Although there is limited IVIVC for nasal permeability models, these models can be used at an air 270 interface to study particle deposition and formulation effects including the functionality and safety of excipients, e.g. by measuring changes in transepithelial electrical resistance induced by absorption enhancers. However, tolerability may vary in vitro vs in vivo as well as in some populations, e.g. pediatrics or older people.

Mucociliary clearance varies within and between people but is a hindrance to drug retention in the nose [30]. In humans, material deposited in the nasal cavity is normally cleared in 12-15 min, although this may rise to $>30 \mathrm{~min}$ if mucociliary 280 clearance is impaired [31]. If a formulation affects drug dissolution or mucus biochemistry and structure, it may modify clearance rate. Experimental models to study mucociliary clearance include ciliated cells for measuring ciliary beat frequency and airway tissue models such as chick and sheep trachea, frog 285 palate. Although the nose is generally regarded as a single compartment in pharmacokinetic modeling, clearance rates may vary from different deposition sites. Mechanistic models are increasingly being used in drug delivery science and can pull together deposition, dissolution, clearance and permeability data in

290 a combinatorial approach which utilize a mix of experimental data and simulations [16,32]. Such an approach could be used to determine the interplay and biological relevance of dissolution and mucociliary clearance.

Device and formulation can be used to target sites in the nasal 295 cavity, e.g. sinuses and NALT which are difficult to reach. Exclusive targeting of such regions seems not possible, but it is possible to influence regional deposition as demonstrated with nasal nebulizers using air vibration to improve deposition in maxillary sinuses $[33,34]$. Furthermore, it must be remembered that dose

300 will redistribute (smear) across the nasal cavity by mucociliary clearance, thus deposition in the turbinates may be adequate to target the nasal mucosal surface for local effects or drug absorption. Validation of targeting specific sites could be achieved by studying regio-temporal drug disposition after applying radiola-

305 belled API to specific nasal sites using a catheter to provide proof of concept for targeting particular absorption sites/mechanisms. Pharmacokinetic-modifying formulation options include polymers, which can modulate absorption and target dendritic cells. Mucus penetrating and mucoadhesive formulations have been

310 studied; some excipients are able to combine both properties, e.g. chitosan [35]. Pharmaceutical acceptability is an important issue for absorption enhancers, although some such as chitosan, alkylsaccharides and pectin are already utilized, in products. Many 'advanced' formulations are under investigation for nose-to-brain 315 delivery, although simple formulations also seem to be effective [36]. However, it remains unclear (i) how much drug reaches the brain via this route, (ii) the nature and capacity of the pathway(s) of the nose-to-brain shunts and their relative contributions to drug transport, and (iii) whether or how nanoparticle formulations enhance drug delivery to the brain via this route. Delivery drugs to the brain may be a benefit or safety concern depending on the drug target.

\section{Conclusions and expert opinion}

The global nasal drug delivery technology market size was valued at USD 41.01 billion in 2017 and is projected to expand $6.5 \%$ by 2025 [37] including novel drugs and targets. New technology and tools will be required to develop these products and key challenges for nasal delivery are how to achieve prolonged nasal retention or target drugs to the systemic circulation or brain selectively (Figure 2). Current research questions include:

- Device innovation Can devices be designed with regard to human factors, including simplicity and robustness? Are such devices able to target specific sites in the nasal cavity?

- Formulation innovation

Can the use of functional excipients be extended (e.g. permeation enhancers; dissolution modifiers) and what are their sensory effects? What new opportunities do nanomedicines offer?

- Critical quality attributes

Can critical attributes be defined for different nasal products, e.g. solution sprays, suspensions, powders or locally versus systemically targeted products? These could be investigated systematically using quality by design approaches.

- Experimental models and simulation

How useful are nasal casts? How can they be 'validated'? How would their usefulness be demonstrated experimentally? Can the nasal vibrissae and mucosal surface be represented in models? Are a range of nasal casts needed (small, medium, large, male/female, children of different ages) to reflect the large variability in anatomy in human subjects?

- Nasal casts for drug deposition

Are current experimental models for dissolution, permeability and clearance fit for testing drug delivery strategies or are new models needed? Can experimental data be used as inputs for mechanistic modelling to predict the pharmacokinetics?

- Nasal delivery in special populations

Are there specific requirements for products for paediatric (infant and child) or elderly patients? How and why should these differ from products for the adult population? Are different devices or formulations needed in patients with nasal pathology?

We offer these questions as a stimulus to research and suggest that they might be addressed most effectively by collaborative research endeavors. Better understanding will enable development of innovative optimized, products for nasal drug delivery and will allow development of evidence-based best practice 


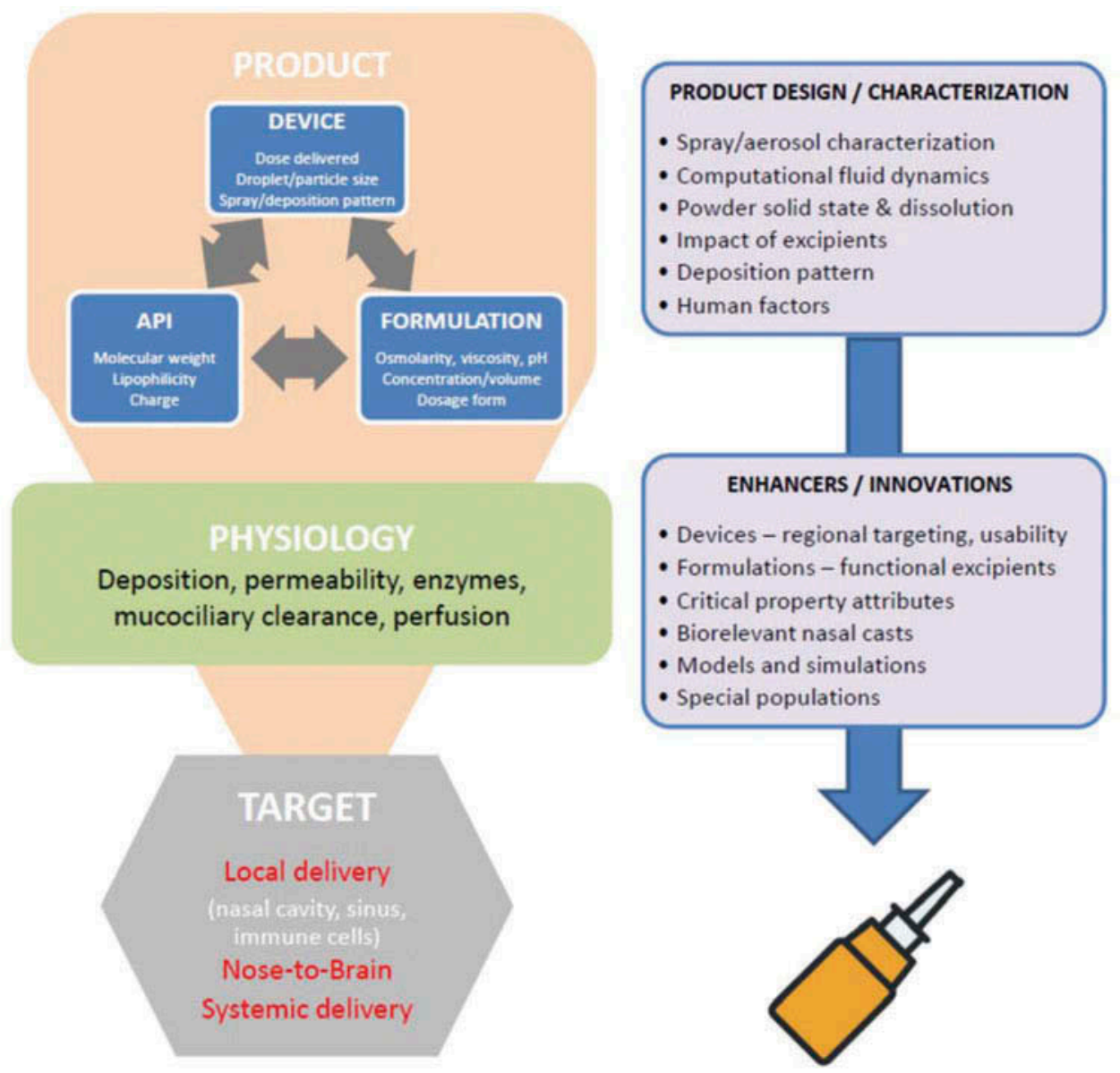

Figure 2. The next generation of nasal products will be required to deliver new classes of molecule, e.g. vaccines, biologics and drugs with action in the brain, to local and systemic therapeutic targets. Innovations and new tools/knowledge are required to design products to deliver these drugs to the right target at the right time in the right patients [38].

and appropriate regulations to assure their quality and fitness for purpose.

\section{Acknowledgments}

R Scherließ, M Hellfritzsch and M Trenkel of Department of Pharmaceutics and Biopharmaceutics, Kiel University, Germany, are thanked for hosting the workshop which forms the basis for this report.

\section{Author Contributions}

This article codifies the gist of the discussions and key issues identified, based on contemporaneous notes made at the meeting; post-hoc referencing benchmarks the subject matter to the pharmaceutical literature. The final expert opinion section of the article reports a consensus on the key research questions for the development of the next generation of nasal drug products. The meeting was designed by B Forbes, R Scherließ and F Sonvico, who created the first draft of the manuscript. All authors attended and contributed to discussion at the workshop. All authors contributed to manuscript review and editing. All authors concur that this report accurately describes the consensus research agenda for optimising nasal drug delivery developed at the workshop.

\section{Funding}

This paper was not funded.

\section{Declaration of Interest}

The authors have no relevant affiliations or financial involvement with any organization or entity with a financial interest in or financial conflict with the subject matter or materials discussed in the manuscript. This includes employment, consultancies, honoraria, stock ownership or options, expert testimony, grants or patents received or pending, or royalties.

\section{Reviewer Disclosures}

Peer reviewers on this manuscript have no relevant financial or other relationships to disclose.

\section{ORCID}

Fabio Sonvico (10) http://orcid.org/0000-0001-7372-1456

Regina Scherließ (D) http://orcid.org/0000-0001-8685-5672

\section{References}

Papers of special note have been highlighted as either of interest $(\cdot)$ or of considerable interest $(\cdot \bullet)$ to readers.

1. Ozsoy $Y$, Gungor $S$, Cevher E. Nasal delivery of high molecular weight drugs. Molecules. 2009;14(9):3754-3779.

2. Hellfritzsch M, Scherließ R. Mucosal vaccination via the respiratory tract. Pharmaceutics. 2019;11:375. 
3. Sonvico F, Clementino A, Buttini F, et al. Surface-modified nanocarriers for nose-to-brain delivery: from bioadhesion to targeting. Pharmaceutics. 2018;10:34.

4. Quintana DS, Westlye LT, Rustan $\varnothing \mathrm{G}$, et al. Low-dose oxytocin delivered intranasally with breath powered device affects social-cognitive behavior: a randomized fourway crossover trial with nasal cavity dimension assessment. Transl Psychiatry. 2015;5:e6.

5. Craft S, Claxtond A, Bakera LD. Effects of regular and long-acting insulin on cognition and Alzheimer's disease biomarkers: a pilot clinical trial. J Alzheimers Dis. 2017;57:1325-1334.

6. Forbes B, Asgharian B, Dailey LA, et al. Challenges in inhaled product development and opportunities for open innovation. Adv Drug Deliv Rev. 2011;63:69-78.

7. FDA. Guidance for industry: nasal spray and inhalation solution, suspension, and spray drug products - chemistry, manufacturing, and controls documentation. 2002. https://www.fda.gov/media/ 70857/download

8. Djupesland PG. Nasal drug delivery devices. Characteristics and performance in a clinical perspective-a review. Drug Deliv Transl Res. 2013;3:42-62.

- Comprehensive review of nasal products and their performance in vivo.

9. Kublik K, Vidgren MT. Nasal delivery systems and their effect on deposition and absorption. Adv Drug Deliv Rev. 1998;29:157-177.

- Albeit twenty years old, this article provides a very broad, overview on nasal drug delivery devices and administration mode as well as formulation impact on nasal deposition and clearance.

10. Costantino HR, Illum L, Brandt G, et al. Intranasal delivery: physicochemical and therapeutic aspects. Int J Pharm. 2007;337:1-24.

11. Grmaš J, Stare K, Bozič D, et al. Elucidation of formulation and delivery device-related effects on in vitro performance of nasal spray with implication to rational product specification identification. J Aerosol Med Pulm Drug Del. 2017;30:230-246.

-. Application of Quality by Design approach to identify device and formulation parameters affecting the in vitro performance of a nasal product.

12. Fasiolo LT, Manniello MD, Tratta E, et al. Opportunity and challenges of nasal powders: drug formulation and delivery. Eur J Pharm Sci. 2018;113:2-17.

- Review of current nasal dry powders formulation strategies and discussion of benefits and challenges of this less orthodox approach to nasal delivery.

13. Salade L, Wauthoz N, Goole J, et al. How to characterize a nasal product. The state of the art of in vitro and ex vivo specific methods. Int J Pharm. 2019;561:47-65.

- Review of current compendial and complementary characterisation techniques being performed in nasal formulation development

14. Salade L, Wauthoz N, Deleu M, et al. Development of coated liposomes loaded with ghrelin for nose-to-brain delivery for the treatment of cachexia. Int J Nanomedicine. 2017;12:S8531-8543.

15. Fransén N, Bredenberg S, Björk E. Clinical study shows improved absorption of desmopressin with novel formulation. Pharm Res. 2009;26:1618-1625.

465 16. Rygg A, Hindle M, Longest PW. Linking suspension nasal spray drug deposition patterns to pharmacokinetic profiles: a proof-of-concept study using computational fluid dynamics. J Pharm Sci. 2016;105:1995-2004.

- An interesting study linking regional nasal spray deposition patterns of suspension formulations, predicted with computational fluid dynamics (CFD), to in vivo human pharmacokinetic (PK) plasma concentration profiles along with compartmental PK modelling.

17. EMA - Guideline on the Pharmaceutical Quality of Inhalation and Nasal Products, 2006
18. Trows S, Wuchner K, Spycher R, et al. Analytical challenges and regulatory requirements for nasal drug products in Europe and the U.S. Pharmaceutics. 2014;6:195-219.

19. FDA. Human Factors Studies and Related Clinical Study Considerations in Combination Product Design and Development. 2016

20. Suman JD, Laube BL, Dalby R. Validity of in vitro tests on aqueous spray pumps as surrogates for nasal deposition, absorption, and biologic response. J Aerosol Med. 2006;19:510-521.

- Seminal paper evidencing how current in vitro performance tests for nasal products may not be clinically relevant and advocating for new approaches for nasal product testing.

21. Liu Q, Absar M, Saluja B, et al. Scientific considerations for the review and approval of first generic mometasone furoate nasal suspension spray in the United States from the bioequivalence perspective. AAPSJ. 2019;21:14

22. Riley T, Christopher D, Arp J, et al. Challenges with developing in vitro dissolution tests for Orally Inhaled Products (OIPs). AAPS PharmSciTech. 2012;13:978-989.

23. Le Guellec S, Le Pennec D, Gatier S, et al. Validation of anatomical models to study aerosol deposition in human nasal cavities. Pharm Res. 2014;31:228-237.

24. Lim ST, Forbes B, Brown MB, et al. Physiological factors affecting nasal drug delivery. Chapter 19. In: Touitou E, Barry BW, editors. Enhancement in drug delivery. Boca Raton: CRC Press; 2007. p. 355-372.

25. Grainger C, Greenwell LL, Lockley DJ, et al. Culture of Calu-3 cells at the air-liquid interface provides a representative model of the airway epithelial barrier. Pharm Res. 2006;23:1482-1490.

26. Pozzoli M, Ong HX, Morgan L, et al. Application of RPMI 2650 nasal cell model to a 3D printed apparatus for the testing of drug deposition and permeation of nasal products. Eur J Pharm Biopharm. 2016;107:223-233.

27. Ong HX, Jackson CL, Cole $\mathrm{JL}$, et al. Primary air-liquid interface culture of nasal epithelium for nasal drug delivery. Mol Pharm. 2016;13:2242-2252.

28. Mercier C, Jacqueroux E, He Z, et al. Pharmacological characterization of the 3D MucilAir ${ }^{\mathrm{TM}}$ nasal model. Eur J Pharm Biopharm. 2019;139:186-196.

29. Borgmann-Winter K, Willard SL, Sinclair D, et al. Translational potential of olfactory mucosa for the study of neuropsychiatric illness. Transl Psychiatry. 2015;5(3):e527-e527.

30. Sveinbjörn G. The effect of cilia and the mucociliary clearance on successful drug delivery. Biol Pharm Bull. 2015;38:497-506.

31. Quadir M, Zia H, Needham TE. Toxicological implications of nasal formulations. Drug Deliv. 1999;6:227-242.

32. Boger Elin FM. Physiologically based pharmacokinetic/pharmacodynamic modeling accurately predicts the better bronchodilatory effect of inhaled versus oral salbutamol dosage forms. J Aerosol Med Pulm Drug Del. 2019;32:1-12.

33. Möller W, Schuschnig U, Khadem Saba G, et al. Pulsating aerosols for drug delivery to the sinuses in healthy volunteers. Otolaryngol Head Neck Surg. 2010;142:382-388.

34. Durand M, Le Guellec S, Pourchez J, et al. Sonic aerosol therapy to target maxillary sinuses. Eur Ann Otorhinolaryngol Head Neck Dis. 2012;129:244-250.

35. Casettari L, Illum L. Chitosan in nasal delivery systems for therapeutic drugs. J Control Release. 2014;190:189-200.

36. Wingrove J, Swedrowska M, Scherließ R, et al. Characterisation of nasal devices for delivery of insulin to the brain and evaluation in humans using functional magnetic resonance imaging. J Control Release. 2019;302:140-147.

37. Grand View Research. Report ID: GVR-2-68038-740-7, Jan 2019. Cited 2019 Sep 06. Available from: https://www.grandviewresearch.com/ industry-analysis/nasal-drug-delivery-technology-system-market

38. Morgan P, Brown DG, Lennard S, et al. Impact of a five-dimensional framework on R\&D productivity at AstraZeneca. Nat Rev Drug Discov. 2018;17:167-181.
480 